\title{
Modeling of organic light emitting diodes: from molecular to device properties
}

\author{
Denis Andrienko
}

\begin{abstract}
We review the progress in modeling of charge transport in disordered organic semiconductors on various lengthscales, from atomistic to macroscopic. This includes evaluation of charge transfer rates from first principles, parametrization of coarse-grained lattice and off-lattice models, and solving the master and drift-diffusion equations. Special attention is paid to linking the length-scales and improving the efficiency of the methods. All techniques are illustrated on an amorphous organic semiconductor, DPBIC, a hole conductor and electron blocker used in state of the art organic light emitting diodes (OLEDs). The outlined multiscale scheme can be used to predict OLED properties without fitting parameters, starting from chemical structures of compounds.
\end{abstract}

Index Terms - organic light emitting diodes, computer simulations

\section{INTRODUCTION}

D esigning new organic materials is crucial for tuning OLED properties, performance, and stability. Rigorous links between device characteristics and the chemical composition of the layers, or structure-property relationships can be provided with the help of cheminformatics tools, i.e., by correlating available experimental data to underlying chemical structures [1]. However, the number of experimental samples is normally limited, since synthesis, device optimization, and characterization are costly and time consuming. A substantial extension of the available data set is therefore not feasible to achieve experimentally, motivating the development of computer simulations techniques capable of predicting the current-voltage-luminescence characteristics of the multilayered OLED structure.

A comprehensive OLED model should incorporate a number of elementary processes: charge injection from electrodes, charge trapping and transport to the emission layer, longrange electron-hole interactions, formation of a charge transfer and excited states, exciton-exciton and exciton-polaron interactions, and radiative decay of excitons. Since the typical thickness of an organic layer of an OLED stack ranges from 10 to $100 \mathrm{~nm}$, it is computationally prohibitive to use only first principles methods for OLED modeling. As a result, state of the art OLED simulations employ either continuous models, such as drift-diffusion equations, or solve the master equation for charge/exciton occupation probabilities. Both approaches use models of different complexity. For example, if the target is to optimize the composition of the stack made of wellcharacterized organic layers, drift-diffusion equations and lattice models with phenomenologically-fitted parameters are

Max Planck Institute for Polymer Research, Ackermannweg 10, Mainz 55128, Germany

Email: denis.andrienko@mpip-mainz.mpg.de used. If one also needs to retain the link to the underlying chemical composition of layers, off-lattice models based on first principles parametrizations are required. In all cases we are dealing with a typical multiscale problem, which requires the development of scale-bridging techniques.

A frequently used ansatz incorporates simulations of atomistic morphologies using classical force-fields, evaluation of charge/exciton transfer rates with first principles methods, and a subsequent analysis of the solution of the master equation [2]-[9]. This modeling is, however, computationally demanding: a layer of a few hundrer nanometers thickness requires simulation boxes of $\sim 10^{7}$ atoms, even if periodic boundary conditions are used in the directions perpendicular to the applied field.

To remedy the situation, extensions to this scheme have been proposed, as depicted in fig. 1. The most straightforward solution is to parametrize a lattice model by matching certain macroscopic properties of the atomistic and lattice models. For example, if charge carrier mobility is the property of interest, one can use the parametric dependencies provided by the family of Gaussian disorder models. This strategy, however, has drawbacks. First, it relies on approximations of the underlying models. Second, the model parametrization is usually performed in stationary conditions. It is therefore not immediately obvious that the same model can be used to describe non-equilibrium (transient) properties of the system.

An alternative approach is to construct an off-lattice model by matching mesoscopic system properties, such as distributions of molecular positions and orientations, electronic couplings, and site energies [10]-[13]. Once parametrized, this model will not require explicit simulations of atomistic morphologies and quantum-chemical evaluations of rates and can therefore be used to simulate large systems. As a result, material properties such as density, radial distribution functions, ionization potentials and electron affinities, energetic disorder, charge mobility and eventually current-voltage characteristics can be extracted from simulations.

In order to achieve a parameter-free modeling of realistic devices, advancements in several directions are still required, among others: first-principles evaluations of charge injection rates, explicit treatment of the induction interaction when solving the master equation, quantitative treatment of excited states embedded in a heterogeneous polarizable molecular environment, descriptions of charge-exciton and exciton-exciton interactions, and descriptions of TTA and TPQ as longerrange Förster and Dexter-type interactions. Progress in these directions will be also reviewed. 


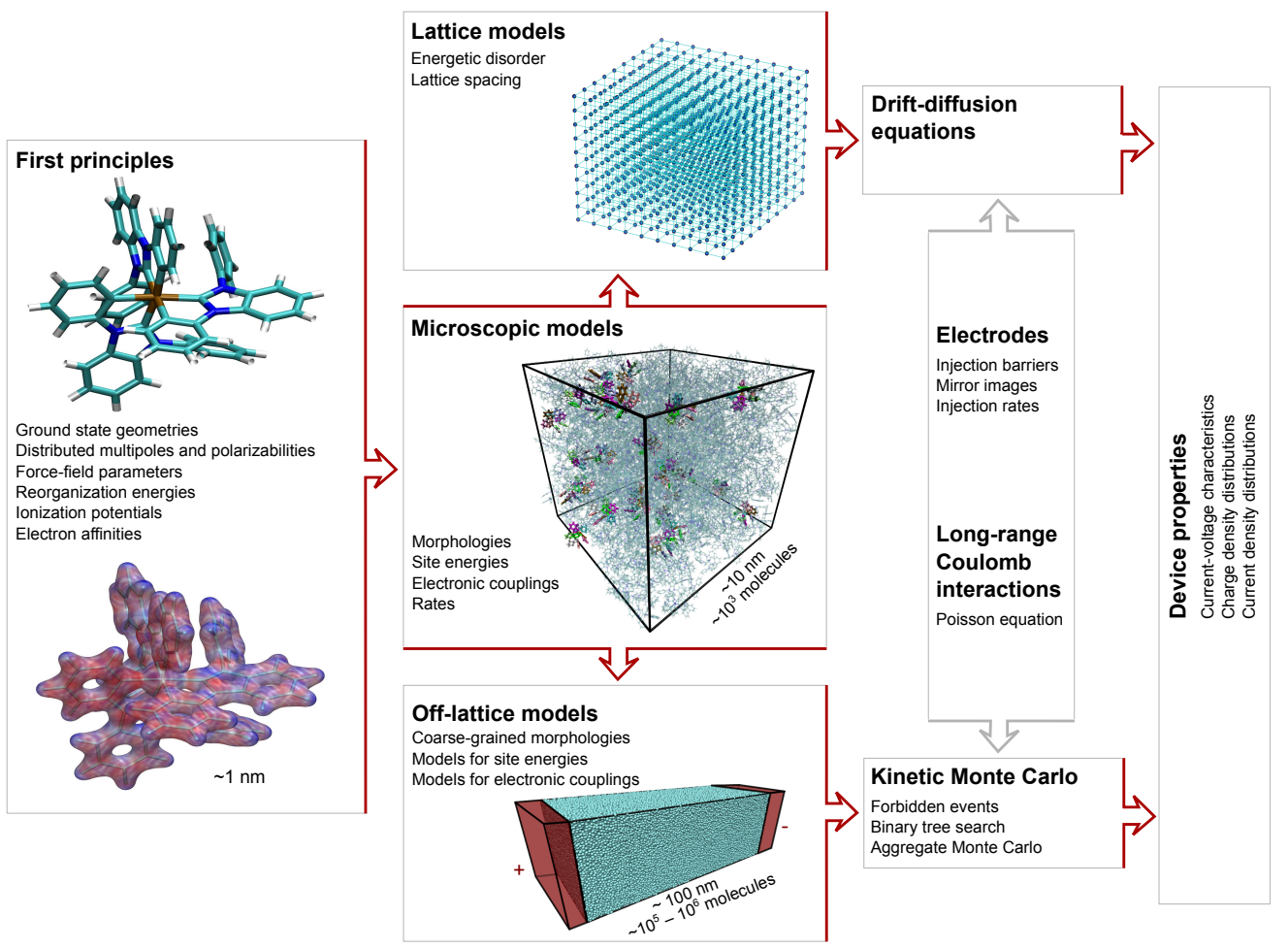

Fig. 1. Possible workflows of parameter-free OLED simulations: polarizable force-fields and electronic properties of isolated molecules obtained from first principles are used to generate amorphous morphologies and evaluate charge transfer rates in small systems. Coarse-grained models are parametrized either by matching macroscopic observables, e.g., charge mobility, of the microscopic and coarse-grained models. The resulting expressions for mobility are then used to solve drift-diffusion equations, after incorporating long-range electrostatic effects and electrodes. Alternatively, off-lattice models can be developed by matching distributions and correlations of site energies, electronic couplings, and molecular positions. The master equation for this model can be solved using the kinetic Monte Carlo algorithm, yielding macroscopic characteristics of a device. Reprinted with permission from Adv. Funct. Mater., 25, 1955 (2015).

\section{ACKNOWLEDGMENT}

This work was in part supported by the BMBF grant InterPhase (FKZ 13N13661) and received funding from the NMP-20-2014 - "Widening materials models" program under Grant Agreement No. 646259 (MOSTOPHOS).

\section{REFERENCES}

[1] T. Bereau, D. Andrienko, and K. Kremer, "Computational materials discovery in soft matter," APL Mat., vol. 4, p. 053101, 2016.

[2] J. Kirkpatrick, V. Marcon, J. Nelson, K. Kremer, and D. Andrienko, "Charge mobility of discotic mesophases: A multiscale quantum and classical study," Phys. Rev. Lett., vol. 98, no. 22, p. 227402, May 2007.

[3] V. Rühle, A. Lukyanov, F. May, M. Schrader, T. Vehoff, J. Kirkpatrick, B. Baumeier, and D. Andrienko, "Microscopic simulations of charge transport in disordered organic semiconductors," J. Chem. Theory Comput., vol. 7, no. 10, pp. 3335-3345, Oct. 2011.

[4] M. Schrader, R. Fitzner, M. Hein, C. Elschner, B. Baumeier, K. Leo, M. Riede, P. Baeuerle, and D. Andrienko, "Comparative study of microscopic charge dynamics in crystalline acceptor-substituted oligothiophenes," J. Am. Chem. Soc., vol. 134, no. 13, pp. 6052-6056, Apr. 2012.

[5] M. Schrader, C. Körner, C. Elschner, and D. Andrienko, "Charge transport in amorphous and smectic mesophases of dicyanovinyl-substituted oligothiophenes," J. Mater. Chem., vol. 22, no. 41, p. 22258, 2012.

[6] F. May, M. Al-Helwi, B. Baumeier, W. Kowalsky, E. Fuchs, C. Lennartz, and D. Andrienko, "Design rules for charge-transport efficient host materials for phosphorescent organic light-emitting diodes," J. Am. Chem. Soc., vol. 134, no. 33, pp. 13 818-13 822, Aug. 2012.

[7] F. May, B. Baumeier, C. Lennartz, and D. Andrienko, "Can lattice models predict the density of states of amorphous organic semiconductors?" Phys. Rev. Lett., vol. 109, no. 13, p. 136401, Sep. 2012.
[8] G. D'Avino, L. Muccioli, F. Castet, C. Poelking, D. Andrienko, Z. Soos, J. Cornil, and D. Beljonne, "Electrostatic phenomena in organic semiconductors: Fundamentals and implications for photovoltaics," Journal of Physics: Condensed Matter, vol. 28, p. 433002, 2016.

[9] C. Poelking and D. Andrienko, "Long-range embedding of molecular ions and excitations in a polarizable molecular environment," J. Chem. Theory Comput., vol. 12, pp. 4516-4523, 2016.

[10] P. Kordt, O. Stenzel, B. Baumeier, V. Schmidt, and D. Andrienko, "Parametrization of extended gaussian disorder models from microscopic charge transport simulations," J. Chem. Theory Comput., vol. 10, no. 6, pp. 2508-2513, Jun. 2014.

[11] O. Stenzel, C. Hirsch, V. Schmidt, T. Brereton, D. Kroese, B. Baumeier, and D. Andrienko, "A general framework for consistent estimation of charge transport properties via random walks in random environments," Multiscale Model. Simul., vol. 12, pp. 1108-1134, 2014.

[12] B. Baumeier, O. Stenzel, C. Poelking, D. Andrienko, and V. Schmidt, "Stochastic modeling of molecular charge transport networks," Phys. Rev. B, vol. 86, no. 18, p. 184202, Nov. 2012.

[13] P. Kordt and D. Andrienko, "Modeling of spatially correlated energetic disorder in organic semiconductors," J. Chem. Theory Comput., vol. 12, pp. 36-40, 2016.

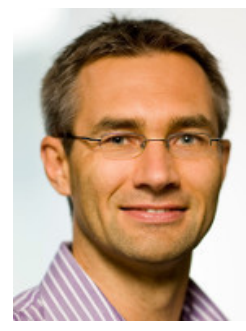

Denis Andrienko Denis Andrienko is a project leader at the Max Planck Institute for Polymer Research working on the development of multiscale simulation techniques for charge and exciton transport in conjugated polymers as well as small molecular weight organic semiconductors. After completing his Masters degree in the University of Kiev he obtained his $\mathrm{PhD}$ on computer simulations of complex fluids from the University of Bristol, UK (group of Prof. M. P. Allen). Dr. Andrienko has published over hundread journal articles and four book chapters. 